\title{
Controlled Self-Assembly of Periodic and Aperiodic Cluster Crystals
}

\author{
Kobi Barkan, ${ }^{1}$ Michael Engel, ${ }^{2}$ and Ron Lifshitz ${ }^{1,3, *}$ \\ ${ }^{1}$ Raymond and Beverly Sackler School of Physics and Astronomy, Tel Aviv University, Tel Aviv 69978, Israel \\ ${ }^{2}$ Department of Chemical Engineering, University of Michigan, Ann Arbor, Michigan 48109, USA \\ ${ }^{3}$ Condensed Matter Physics 149-33, California Institute of Technology, Pasadena, California 91125, USA
}

(Received 16 January 2014; published 28 August 2014)

\begin{abstract}
Soft particles are known to overlap and form stable clusters that self-assemble into periodic crystalline phases with density-independent lattice constants. We use molecular dynamics simulations in two dimensions to demonstrate that, through a judicious design of an isotropic pair potential, one can control the ordering of the clusters and generate a variety of phases, including decagonal and dodecagonal quasicrystals. Our results confirm analytical predictions based on a mean-field approximation, providing insight into the stabilization of quasicrystals in soft macromolecular systems, and suggesting a practical approach for their controlled self-assembly in laboratory realizations using synthesized soft-matter particles.
\end{abstract}

Particles interacting via pair potentials with repulsive cores, which are either bounded or only slowly diverginglike those found naturally in soft matter systems [1] — can be made to overlap under pressure to form clusters [2], which then self-assemble to form crystalline phases [3]. The existence of such cluster crystals was recently confirmed in amphiphilic dendritic macromolecules using monomer-resolved simulations [4], and in certain bosonic systems [5]. They occur even when the particles are purely repulsive, and typically exhibit periodic fcc or bcc structures. Here we employ molecular dynamics (MD) simulations in two dimensions, guided by analytical insight, to show how isotropic pair potentials can be designed to control the self-assembly of the clusters, suggesting a practical approach that could be applied in the laboratory. We obtain novel phases, including a striped (lamellar) phase and a hexagonal superstructure, as well as decagonal (tenfold) and dodecagonal (twelvefold) quasicrystals.

Given a system of $N$ particles in a box of volume $V$, interacting via an isotropic pair potential $U(r)$ with a repulsive core, a sufficient condition for the formation of a cluster crystal is a negative global minimum $\tilde{U}_{\min }=\tilde{U}\left(k_{\min }\right)<0$ in the Fourier transform of the potential [6]. This condition implicitly requires the potential not to diverge too strongly, so that the Fourier transform exists. The wave number $k_{\min }$ determines the length scale for the order in the system by setting the typical distance between neighboring clusters. Above a sufficiently high mean particle density $\bar{c}=N / V$, a further increase of $\bar{c}$ increases the number of overlapping particles within each cluster, but does not change the distance between their centers. It also determines the spinodal temperature $k_{B} T_{\mathrm{sp}}=-\tilde{U}_{\min } \bar{c}$ $[3,6]$, below which the liquid becomes unstable against crystallization, where $k_{B}$ is the Boltzmann constant.

As the particles form increasingly larger clusters, the system becomes well characterized by a continuous coarse-grained density function $c(\boldsymbol{r})$. The thermodynamic behavior can then be described in a mean-field approximation, which becomes exact in the high-density hightemperature limit [7]. Using MD simulations, we examine the analytical predictions of a particular mean-field approximation that was proposed by Barkan, Diamant, and Lifshitz (BDL) [8] to explain the stability of a certain class of soft quasicrystals in two dimensions. BDL confirmed an earlier conjecture [9] that attributed the stability of soft quasicrystals to the existence of two length scales in the pair potential, combined with effective many-body interactions arising from entropy. Accordingly, we study pair potentials whose Fourier transforms contain two negative minima of the same depth $\tilde{U}_{\text {min }}$, like the ones shown in Fig. 1. That stable quasicrystals may need two length scales in their effective interaction potentials is not new [10]. Many two-length-scale potentials were investigated over the years and found to exhibit stable quasiperiodic phases [11]. The novelty of BDL was in their quantitative understanding of the stabilization mechanism, allowing them to pinpoint regions of stability in the parameter spaces of different potentials, instead of performing an exhaustive search. The inclusion of a second length scale provides greater control over the self-assembly of the clusters than what can be achieved with a single minimum only. We demonstrate how this enables one to generate a wide range of novel periodic and aperiodic cluster crystal structures.

Our findings come at a time when an ever-growing number of soft-matter systems are found to exhibit phases with quasiperiodic long-range order-mostly showing dodecagonal symmetry [12]. First discovered in liquid crystals made of amphiphilic dendritic macromolecules [13], self-assembled soft quasicrystals have since appeared in $A B C$-star polymers [14], in sphere-forming block 


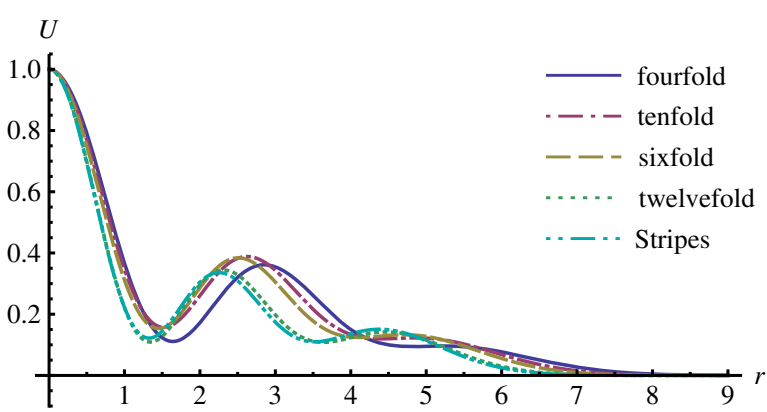

(a)

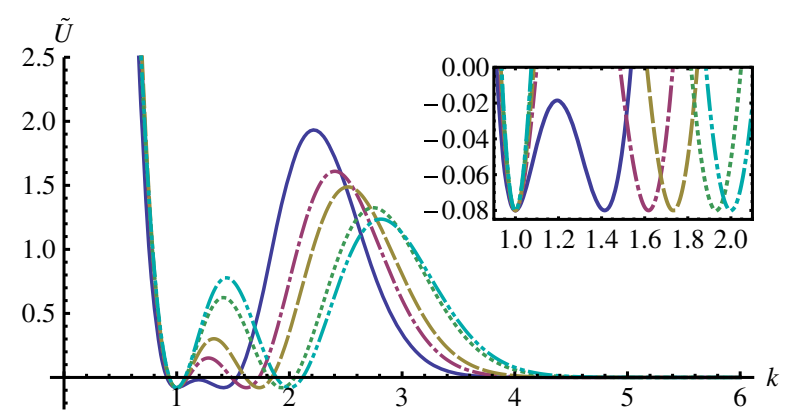

(b)

FIG. 1 (color online). Pair potentials used in this study. (a) Real space $U(r)$, normalized such that $U(0)=1$. (b) Fourier transform $\tilde{U}(k)$, where the first minimum is always at $k=1$, and the second minimum is located with increasing order at $k_{4}=\sqrt{2}$ (solid blue), $k_{5}=(1+\sqrt{5}) / 2$ (dot-dashed red), $k_{6}=\sqrt{3}$ (dashed brown), $k_{12}=\sqrt{2+\sqrt{3}}$ (dotted green), and $k_{\infty}=2$ (double-dot-dashed cyan). The inset shows a close-up view of the minima.

copolymers [15], in systems of nanoparticles [16], with anisotropic particles [17] and pentameric molecules [18], and in mesoporous silica [19]. These systems provide exciting platforms for the fundamental study of the physics of quasicrystals [20] and promise new applications of self-assembled nanomaterials [21].

The key idea of BDL was borrowed from Lifshitz and Petrich (LP) [22], who extended the Swift-Hohenberg equation [23] to study parametrically excited surface waves (Faraday waves), also exhibiting dodecagonal quasiperiodic order [24]. The Swift-Hohenberg equation is a generic model for pattern-forming systems [25] that describes the instability of a uniform state against the formation of Fourier modes with a fixed and finite wave number. In the LP modification the instability occurs simultaneously at two wave numbers, whose ratio $q$ is tunable. It is then the role of resonant three-mode interactions to stabilize structures containing triplets of Fourier modes with wave vectors that add up to zero. By setting the value of the wave-number ratio $q$ to $k_{n} \equiv 2 \cos (\pi / n)$, one can form triplets containing two unit wave vectors separated by $2 \pi / n$, and a third wave vector of length $k_{n}$. Indeed, stable patterns with $n$-fold symmetry were shown to exist in the LP model for $n=4,6$, and 12, with wave-number ratios $k_{4}=\sqrt{2}, k_{6}=\sqrt{3}$, and $k_{12}=\sqrt{2+\sqrt{3}}$, respectively, as well as stripes for $k_{\infty}=2$ [22]. Patterns with eightfold symmetry are unstable within the LP model, but there is a narrow window of stability for tenfold patterns with a ratio of $k_{5}=(1+\sqrt{5}) / 2$, although not with $k_{10}=\sqrt{(5+\sqrt{5}) / 2}[26]$.

Based on these design principles, and to remain as pedagogical as possible, we work directly in Fourier space to construct the family of smooth pair potentials shown in Fig. 1. Yet, we emphasize that our approach can be applied to any realistic potential with sufficiently many tunable parameters. We use a polynomial in even powers of the wave number $k$, such that two equal-depth minima can explicitly be positioned at 1 and $q=k_{n}$, similar in form to the effective potential used by LP. We then multiply this polynomial by a Gaussian to limit the extent of the potential. These LP-Gaussian potentials are given in Fourier space by

$$
\tilde{U}(k)=e^{-k^{2} / 2 \sigma^{2}}\left(D_{0}+D_{2} k^{2}+D_{4} k^{4}+D_{6} k^{6}+D_{8} k^{8}\right),
$$

and are self-dual in the sense that they have the same functional form in real space. Using a two-dimensional Fourier transform we obtain

$$
\begin{aligned}
U(r) & =\frac{1}{2 \pi} \int_{0}^{\infty} \tilde{U}(k) J_{0}(k r) k d k \\
& =e^{-\sigma^{2} r^{2} / 2}\left(C_{0}+C_{2} r^{2}+C_{4} r^{4}+C_{6} r^{6}+C_{8} r^{8}\right),
\end{aligned}
$$

where $J_{0}$ is the zeroth-order Bessel function, and the polynomial coefficients $C_{0}, \ldots, C_{8}$ are linear functions of the Fourier-space coefficients $D_{0}, \ldots, D_{8}$. We set the latter five independent coefficients such that $U(0)=C_{0}=1$, and there are two equal-depth minima at positions 1 and $k_{n}$ in reciprocal space with $\tilde{U}(1)=\tilde{U}\left(k_{n}\right)=-0.08$ [27]. This sets both the energy scale and the length scale in our description of the problem. The standard deviation $\sigma$ of the Gaussian in reciprocal space is chosen such that the potentials are purely repulsive in real space, although this is not required. Note that while it is difficult to tell the potentials apart in real space [Fig. 1(a)] and therefore not obvious to anticipate which cluster crystal they will stabilize, the potentials are clearly distinguishable in reciprocal space [Fig. 1(b)], where the wave-number ratio $q$ is visible. Similarly, one would need to tune the realspace parameters of any realistic potential to possess the required minima in Fourier space. The LP-Gaussian potentials benefit from being simple, bounded, and rapidly decaying and therefore amenable to MD simulations.

We initialize GPU-accelerated MD simulations [29] in the liquid phase above the melting temperature. The system is slowly cooled down in the $N V T$ ensemble to induce self-assembly. At $T=0$, the protocol is reversed until melting occurs. Typically, the first signs of ordering are 


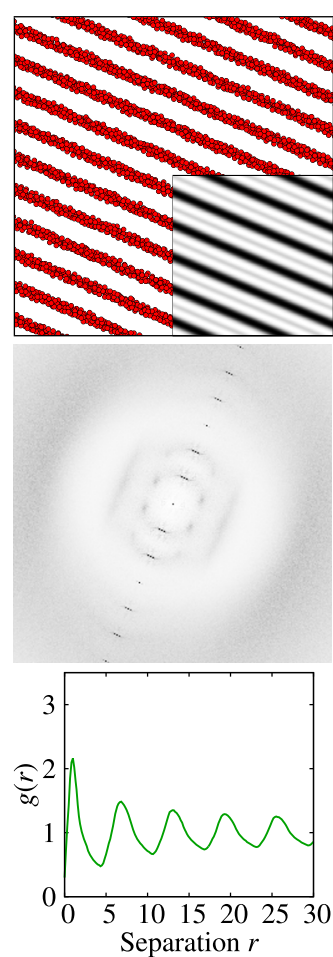

(a) $k_{\infty}=2$
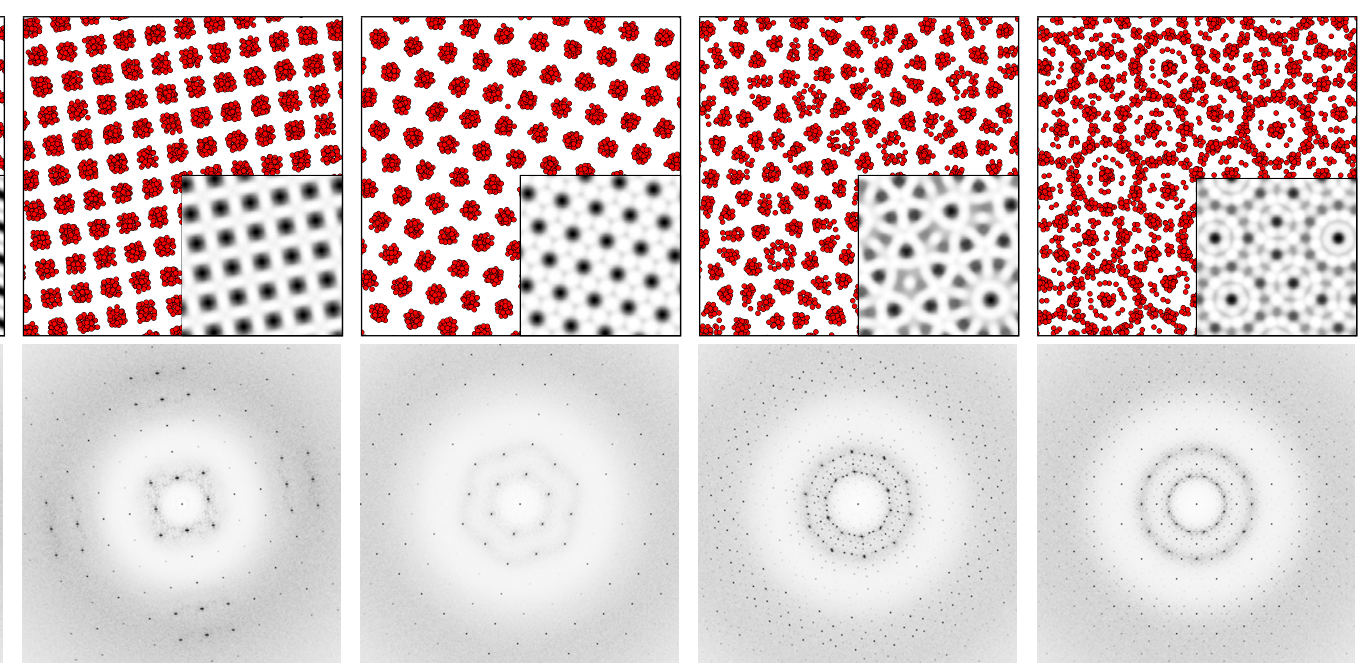

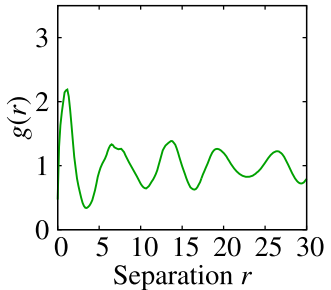

(b) $k_{4}=\sqrt{2}$

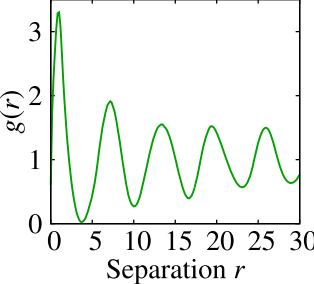

(c) $k_{6}=\sqrt{3}$

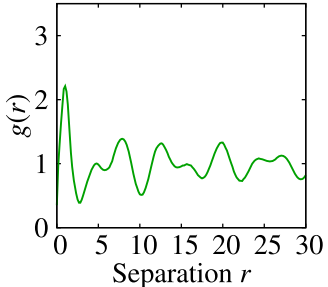

(d) $k_{5}=(1+\sqrt{5}) / 2$

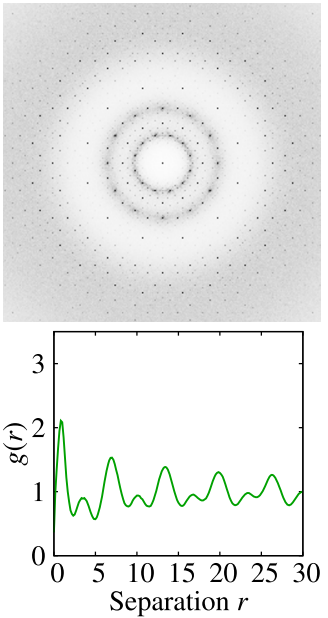

(e) $k_{12}=\sqrt{2+\sqrt{3}}$

FIG. 2 (color online). Self-assembled cluster crystals for the pair potentials in Fig. 1. We generate (a) stripes, periodic (b) tetragonal, and (c) hexagonal, as well as quasiperiodic (d) decagonal and (e) dodecagonal cluster crystals. Simulation parameters are $N=16384, T=0.03$, and $\bar{c}=0.8$ (a),(e), $\bar{c}=0.9$ (b), $\bar{c}=0.7$ (c), $\bar{c}=0.6$ (d). The top, middle, and bottom rows show snapshots in real space comparing MD results (red circles) with mean-field predictions for $c(\boldsymbol{r})$ (in gray scale at the bottom-right corners), diffraction diagrams, and radial distribution functions. Particles are drawn with radius 1 . The real-space view is limited to about $20 \%$ of the simulation box [27].

strong density fluctuations in the liquid, which then condense into clusters and spread to develop global order. Individual particles can migrate between neighboring clusters at elevated temperatures, even after cluster crystallization has set in, to average out density fluctuations and heal defects. We observe self-assembly at all densities in the studied range $0.1 \leq \bar{c} \leq 2.0$. While at low densities, below $\bar{c} \approx 0.5$, the particles behave more individually and the hexagonal crystal prevails, at higher densities the MD results exactly match the equilibrium structures predicted by mean-field theory, as demonstrated in the top row of Fig. 2. As expected, we find striped (lamellar), tetragonal, hexagonal, decagonal, and dodecagonal cluster crystals. In all cases, the strongest peaks in the diffraction diagrams are located at 1 and $k_{n}$, the two minima of $\tilde{U}(k)$, followed by a ring with little scattering where $\tilde{U}(k)$ has its maximum. The superstructures formed by thinner stripes within the striped phase and smaller clusters within the hexagonal phase, predicted by the mean-field densities in Figs. 2(a) and 2(c), are observed in the MD simulations at higher densities [27]. The hexagonal superstructure is analogous to the superlattice structures observed in Faraday waves [30].

The transition from the liquid to a cluster crystal is a first order phase transition and therefore accompanied by hysteresis. We use "error" bars in Fig. 3 to show the temperature range of coexistence, obtained from simulation, as a function of $\bar{c}$. The bars span the temperature range from where crystallization is observed upon cooling to where melting occurs upon heating. The lower ends are bounded from below by the mean-field predicted $T_{\text {sp }}$, shown in Fig. 3

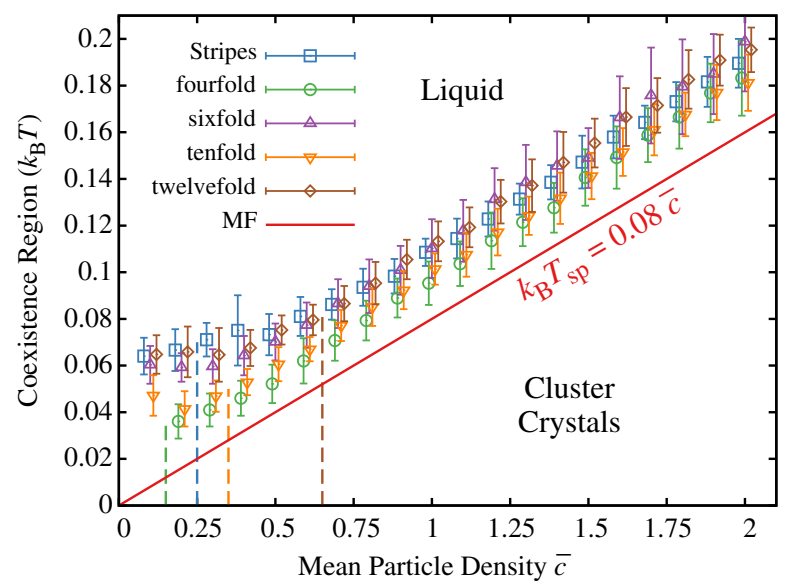

FIG. 3 (color online). Coexistence of the liquid and cluster crystal phases, shown as bars, as a function of mean particle density $\bar{c}$ from simulation data. The red line is the mean-field spinodal (sp) instability limit. Cluster crystals with $n$-fold rotational symmetry are observed exclusively for $\bar{c}$ above the vertical dashed lines, while hexagonal cluster crystals are found below. Densities are slightly shifted horizontally by \pm 0.01 among different cluster crystals for better visibility. 

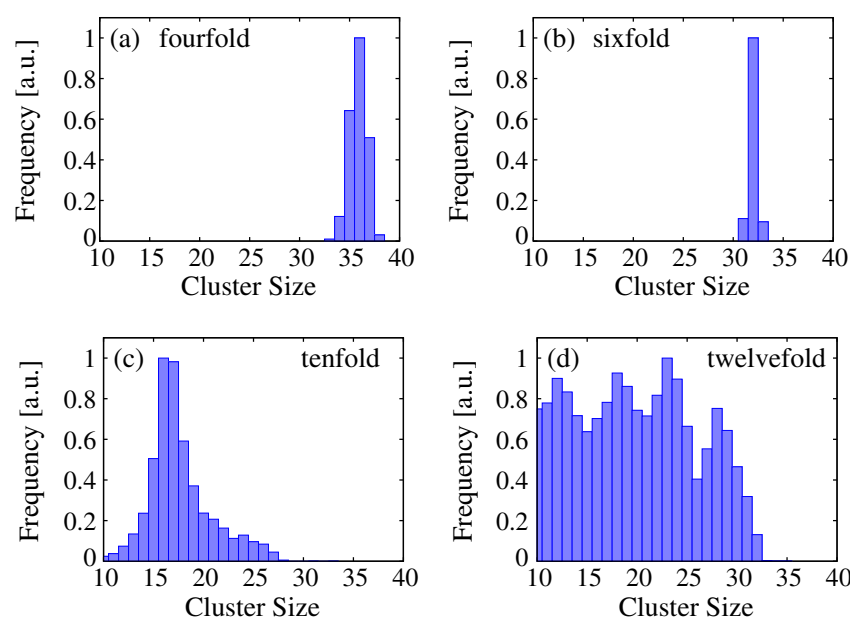

FIG. 4 (color online). Histograms of the cluster sizes in Fig. 2. (a),(b) Periodic crystals show sharp distributions. (c),(d) Quasicrystals exhibit broad distributions. We use the cluster size cutoff parameter MinPts $=8$ for the DBSCAN algorithm [31].

as a straight line. Except for low densities, where the meanfield approximation fails, we observe a shift of about $0.01-0.03$ between the simulated freezing points and the mean-field spinodal line. This shift is due to thermal fluctuations causing the cooled liquid to become unstable and freeze earlier.

We further characterize the ordered phases by identifying individual clusters using the DBSCAN algorithm [31]. Figure 4 demonstrates that while the cluster size distribution is narrowly peaked for periodic cluster crystals indicating a single characteristic cluster size, the cluster size distribution has a broad peak for the decagonal cluster crystal and is flat and almost featureless for the dodecagonal cluster crystal. This observation is in agreement with experimentally observed distributions of high-symmetry stars in quasiperiodic light fields [32] and with the meanfield density profiles shown alongside the MD simulation results in Fig. 2.

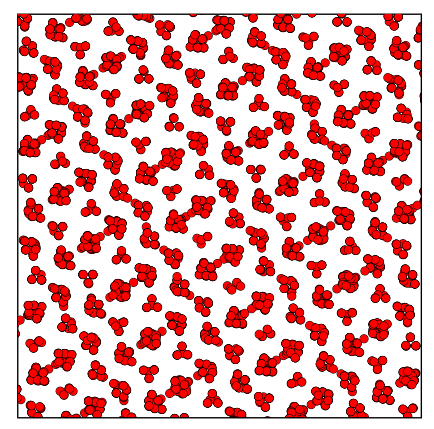

(a) $k_{12}: \bar{c}=1.9, k_{\mathrm{B}} T=10^{-3}$

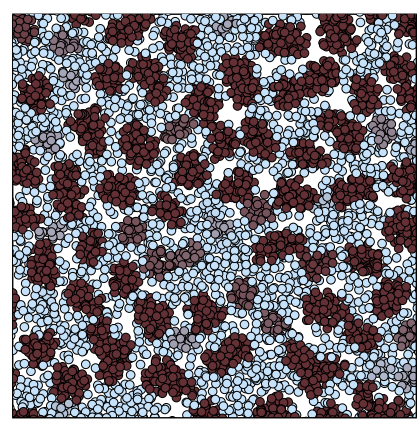

(b) $k_{4}: \bar{c}=2.0, k_{\mathrm{B}} T=0.18$
FIG. 5 (color online). (a) At low temperature and high density the dodecagonal quasicrystal can reversibly transform into a compressed hexagonal phase [27]. (b) Snapshot of the liquid phase at a temperature just above the onset of ordering, possibly showing a liquid of clusters. Clusters are colored according to their size from small (light blue, size $\leq 10$ ) to large (dark red, size $\geq 30$ ).
At lower temperatures, mean-field theory predicts that the quasicrystals should become unstable toward a secondary transformation into a periodic phase of lower rotational symmetry, such as a hexagonal cluster crystal [8]. We do not observe a transformation for the decagonal quasicrystal. We do observe a secondary transformation for the dodecagonal quasicrystal into either the so-called $\sigma$ phase [27], which is a known periodic approximant for dodecagonal quasicrystals that is commonly observed in soft-matter systems [13-16], or a compressed hexagonal phase [Fig. 5(a)], similar to the one considered by LP in their Fig. 2(b). In all cases we find that the transformation is reversible and the quasicrystal reforms in simulation upon heating, confirming the mean-field prediction regarding the role of entropy in its stabilization. We frequently observe significant density fluctuations already prior to ordering, as shown in Fig. 5(b), indicating the possibility that first a cluster liquid is formed, and then the clusters order. This opens up interesting questions about the formation mechanism of the cluster crystals. The observation that cluster crystals sometimes "lock-in" their orientation to the simulation box [33] suggests that fluctuations are important and classical nucleation theory might not be applicable.

To conclude, we have shown how to control the selfassembly of a variety of cluster crystals by using isotropic pair potentials with two length scales, and designing their ratio in Fourier space-a general procedure that can be applied to other kinds of potentials and in the lab, and which expands upon earlier potential design schemes [34]. This work can be continued in several directions. Longer and larger simulations, accompanied by numerical freeenergy calculations [35], are necessary to obtain more precise phase diagrams and to accurately identify the stability regions and their dependence on cooling rates and finite size effects. The dynamics leading to crystallization and the study of collective phonon and phason degrees of freedom in the ordered state are open problems. Finally, an extension to three dimensions is a next step toward making a firmer contact with experimental observations of quasicrystals in soft matter systems.

We are grateful to Michael Cross, Haim Diamant, Jens Glaser, Liron Korkidi, and Goran Ungar for fruitful discussions. This research is supported by the Israel Science Foundation through Grant No. 556/10.

* Corresponding author. ronlif@tau.ac.il

[1] C. N. Likos, Phys. Rep. 348, 267 (2001).

[2] W. Klein, H. Gould, R. A. Ramos, I. Clejan, and A. I. Melćuk, Physica (Amsterdam) 205A, 738 (1994); C. N. Likos, M. Watzlawek, and H. Löwen, Phys. Rev. E 58, 3135 (1998).

[3] F. H. Stillinger, J. Chem. Phys. 65, 3968 (1976); C. N. Likos, N. Hoffmann, H. Löwen, and A. A. Louis, J. Phys. Condens. Matter 14, 7681 (2002); B. M. Mladek, D. Gottwald, G. Kahl, M. Neumann, and C. N. Likos, Phys. 
Rev. Lett. 96, 045701 (2006); K. Zhang, P. Charbonneau, and B. M. Mladek, Phys. Rev. Lett. 105, 245701 (2010).

[4] D. A. Lenz, R. Blaak, C. N. Likos, and B. M. Mladek, Phys. Rev. Lett. 109, 228301 (2012).

[5] F. Cinti, P. Jain, M. Boninsegni, A. Micheli, P. Zoller, and G. Pupillo, Phys. Rev. Lett. 105, 135301 (2010); N. Henkel, F. Cinti, P. Jain, G. Pupillo, and T. Pohl, Phys. Rev. Lett. 108, 265301 (2012).

[6] C. N. Likos, A. Lang, M. Watzlawek, and H. Löwen, Phys. Rev. E 63, 031206 (2001); C. N. Likos, B. M. Mladek, D. Gottwald, and G. Kahl, J. Chem. Phys. 126, 224502 (2007); B. M. Mladek, D. Gottwald, G. Kahl, M. Neumann, and C. N. Likos, J. Phys. Chem. B 111, 12799 (2007).

[7] A. Lang, C. N. Likos, M. Watzlawek, and H. Löwen, J. Phys. Condens. Matter 12, 5087 (2000); A. A. Louis, P. G. Bolhuis, and J. P. Hansen, Phys. Rev. E 62, 7961 (2000).

[8] K. Barkan, H. Diamant, and R. Lifshitz, Phys. Rev. B 83, 172201 (2011).

[9] R. Lifshitz and H. Diamant, Philos. Mag. 87, 3021 (2007).

[10] Z. Olami, Phys. Rev. Lett. 65, 2559 (1990); A. P. Smith, Phys. Rev. B 43, 11635 (1991).

[11] M. Dzugutov, Phys. Rev. Lett. 70, 2924 (1993); E. A. Jagla, Phys. Rev. E 58, 1478 (1998); A. Skibinsky, S. V. Buldyrev, A. Scala, S. Havlin, and H. E. Stanley, Phys. Rev. E 60, 2664 (1999); A. Quandt and M. P. Teter, Phys. Rev. B 59, 8586 (1999); J. Roth and A. R. Denton, Phys. Rev. E 61, 6845 (2000); A. S. Keys and S. C. Glotzer, Phys. Rev. Lett. 99, 235503 (2007); M. Engel and H.-R. Trebin, Phys. Rev. Lett. 98, 225505 (2007); Z. Kristallogr. 223, 721 (2008); J. C. Johnston, N. Kastelowitz, and V. Molinero, J. Chem. Phys. 133, 154516 (2010); A. J. Archer, A. M. Rucklidge, and E. Knobloch, Phys. Rev. Lett. 111, 165501 (2013); T. Dotera, T. Oshiro, and P. Ziherl, Nature (London) 506, 208 (2014); C. V. Achim, M. Schmiedeberg, and H. Löwen, Phys. Rev. Lett. 112, 255501 (2014).

[12] G. Ungar and X. Zeng, Soft Matter 1, 95 (2005); G. Ungar, V. Percec, X. Zeng, and P. Leowanawat, Isr. J. Chem. 51, 1206 (2011); T. Dotera, Isr. J. Chem. 51, 1197 (2011); J. Polym. Sci., Part B: Polym. Phys. 50, 155 (2012).

[13] X. Zeng, G. Ungar, Y. Liu, V. Percec, A. E. Dulcey, and J. K. Hobbs, Nature (London) 428, 157 (2004).

[14] A. Takano, W. Kawashima, A. Noro, Y. Isono, N. Tanaka, T. Dotera, and Y. Matsushita, J. Polym. Sci., Part B: Polym. Phys. 43, 2427 (2005); K. Hayashida, T. Dotera, A. Takano, and Y. Matsushita, Phys. Rev. Lett. 98, 195502 (2007).

[15] S. Fischer, A. Exner, K. Zielske, J. Perlich, S. Deloudi, W. Steurer, P. Lindner, and S. Förster, Proc. Natl. Acad. Sci. U.S.A. 108, 1810 (2011); J. Zhang and F. S. Bates, J. Am. Chem. Soc. 134, 7636 (2012).

[16] D. V. Talapin, E. V. Shevchenko, M. I. Bodnarchuk, X. Ye, J. Chen, and C. B. Murray, Nature (London) 461, 964 (2009); M. I. Bodnarchuk, R. Erni, F. Krumeich, and M. V. Kovalenko, Nano Lett. 13, 1699 (2013).

[17] A. Haji-Akbari, M. Engel, A. S. Keys, X. Zheng, R. G. Petschek, P. Palffy-Muhoray, and S. C. Glotzer, Nature (London) 462, 773 (2009); C. R. Iacovella, A. S. Keys, and S. C. Glotzer, Proc. Natl. Acad. Sci. U.S.A. 108, 20935 (2011); J. Dontabhaktuni, M. Ravnik, and S. Žumer, Proc. Natl. Acad. Sci. U.S.A. 111, 2464 (2014).
[18] N. A. Wasio, R. C. Quardokus, R. P. Forrest, C. S. Lent, S. A. Corcelli, J. A. Christie, K. W. Henderson, and S. A. Kandel, Nature (London) 507, 86 (2014).

[19] C. Xiao, N. Fujita, K. Miyasaka, Y. Sakamoto, and O. Terasaki, Nature (London) 487, 349 (2012).

[20] R. Lifshitz, Isr. J. Chem. 51, 1156 (2011).

[21] V. Percec, M. Glodde, T. K. Bera, Y. Miura, I. Shiyanovskaya, K. D. Singer, V. S. K. Balagurusamy, P. A. Heiney, I. Schnell, A. Rapp, H.-W. Spiess, S. D. Hudson, and H. Duan, Nature (London) 417, 384 (2002); D. K. Smith, A. R. Hirst, C. S. Love, J. G. Hardy, S. V. Brignell, and B. Huang, Prog. Polym. Sci. 30, 220 (2005).

[22] R. Lifshitz and D. M. Petrich, Phys. Rev. Lett. 79, 1261 (1997).

[23] J. B. Swift and P. C. Hohenberg, Phys. Rev. A 15, 319 (1977).

[24] W. S. Edwards and S. Fauve, Phys. Rev. E 47, R788 (1993).

[25] M. Cross and H. Greenside, Pattern Formation and Dynamics in Nonequilibrium Systems (Cambridge University Press, Cambridge, 2009), Chap. 5.

[26] The $k_{12}$ quasicrystal is very robust with its 24 Fourier modes and 32 stabilizing triplets. The $k_{8}$ quasicrystal has 16 modes with only 8 triplets, and the $k_{10}$ quasicrystal has 20 modes with 10 triplets, making them both always unstable with respect to the simple hexagonal phase with its 6 modes and 2 triplets. The $k_{5}$ quasicrystal has 20 modes with 20 triplets, making it just barely stable if parameters are exactly tuned, but it is not as robust as the dodecagonal phase against slight deviations from the exact requirements. We believe that for this reason decagonal quasicrystals have not yet been observed in real systems of soft isotropic particles.

[27] See Supplemental Material at http://link.aps.org/ supplemental/10.1103/PhysRevLett.113.098304, which includes Ref. [28], for additional simulation snapshots and a discussion of potential parameters and methods.

[28] K. Jiang and P. Zhang, J. Comput. Phys. 256, 428 (2014).

[29] J. A. Anderson, C. D. Lorenz, and A. Travesset, J. Comput. Phys. 227, 5342 (2008).

[30] H. Arbell and J. Fineberg, Phys. Rev. E 65, 036224 (2002).

[31] M. Ester, H.-P. Kriegel, J. Sander, and X. Xu, in Proceedings of the Second International Conference on Knowledge Discovery and Data Mining, edited by E. Simoudis, J. Han, and U. M. Fayyad (AAAI Press, 1996) pp. 226-231.

[32] J. Mikhael, M. Schmiedeberg, S. Rausch, J. Roth, H. Stark, and C. Bechinger, Proc. Natl. Acad. Sci. U.S.A. 107, 7214 (2010).

[33] We confirmed the occurrence of the lock-in phenomena, where a main crystallographic axis is oriented parallel to the simulation box, in about $10 \%-20 \%$ of the simulations with $N=16384$ using two independent MD codes.

[34] M. C. Rechtsman, F. H. Stillinger, and S. Torquato, Phys. Rev. Lett. 95, 228301 (2005); H. Cohn and A. Kumar, Proc. Natl. Acad. Sci. U.S.A. 106, 9570 (2009); E. Edlund, O. Lindgren, and M. N. Jacobi, Phys. Rev. Lett. 107, 085503 (2011); A. Jain, J. R. Errington, and T. M. Truskett, J. Chem. Phys. 139, 141102 (2013).

[35] B. M. Mladek, P. Charbonneau, and D. Frenkel, Phys. Rev. Lett. 99, 235702 (2007); M. Engel, Phys. Rev. Lett. 106, 095504 (2011); A. Reinhardt, F. Romano, and J. P. K. Doye, Phys. Rev. Lett. 110, 255503 (2013). 